\title{
Fabrication of Novel Superhydrophobic Surfaces and Droplet Bouncing Behavior - Part 2: Water Droplet Impact Experiment on Superhydrophobic Surfaces Constructed Using ZnO Nanoparticles
}

\author{
Bin-Bin Wang ${ }^{\mathrm{a}}$, Ya-Pu Zhao ${ }^{\mathrm{a}, *}$ and Tongxi Yu ${ }^{\mathrm{b}}$ \\ ${ }^{a}$ State Key Laboratory of Nonlinear Mechanics, Institute of Mechanics, \\ Chinese Academy of Sciences, Beijing 100190, China \\ ${ }^{\mathrm{b}}$ Department of Mechanical Engineering, Hong Kong University of Science and Technology, \\ Clear Water Bay, Kowloon, Hong Kong SAR, China
}

Received in final form 9 March 2010

\begin{abstract}
When a liquid droplet impacts a solid surface, it spreads up to a point and the kinetic energy is dissipated by viscosity, collision and surface energy during the process. The droplet can retract if the energy dissipation during the impact process which is only partly governed by surface properties is not too large. Otherwise, the droplet would stick to the surface or break into smaller droplets. In this second part, we introduced contact angle hysteresis (CAH) and studied the impact behavior between a water droplet and a superhydrophobic surface both theoretically and experimentally. On our superhydrophobic surface, the contact angle is about $155^{\circ}$, so the kinetic energy of the droplet can be largely transferred to surface energy. Thus, under certain conditions, the droplet can fully bounce. The impact behavior of normal impact was analyzed theoretically. The critical falling heights for rebound (CFHR) were investigated on constructed ZnO-PDMS superhydrophobic surface in both normal and oblique impact conditions, and CFHR was found to increase with the increase of tilt angle. This shows that the normal Weber number $\left(W e_{n}\right)$ is the major factor governing the rebound, while the tangential Weber number $\left(W e_{t}\right)$ also has effect on the phenomenon. Compared to the energy dissipated by collision and viscosity, the influence of surface properties is relatively small. The adhesion number $\left(N_{\mathrm{a}}\right)$ is the parameter determining the energy dissipated by surface tension and $N_{\mathrm{a}}$ has direct relation with contact angle (CA) and $\mathrm{CAH}$.
\end{abstract}

(C) Koninklijke Brill NV, Leiden, 2011

\section{Keywords}

Superhydrophobic surface, water droplet, normal impact, oblique impact, contact angle hysteresis, adhesion number, energy dissipation

\footnotetext{
* To whom correspondence should be addressed. Fax: +86-100-6256-1284; e-mail: yzhao@imech.ac.cn
} 


\section{Notations}

$A$ liquid-solid contact area

$\mathrm{Ca}$ capillary number

$d$ diameter of maximal liquid-solid contact area

$d_{0}$ original diameter of droplet

$F_{\mathrm{a}}$ adhesion force

$F_{\text {re }}$ reaction force

$H_{0}$ critical falling height for normal impact

$g$ acceleration of gravity

$k$ stiffness of spring

$K$ constant of proportionality

$l_{0}$ initial length of spring

$m$ mass of droplet

$N_{\mathrm{a}}$ adhesion number

$r$ radius of droplet

$R$ radius of liquid-solid contact area

$R e$ Reynolds number

$t$ time

$V$ velocity of droplet before impact

$V^{\prime}$ velocity of droplet after impact

We Weber number

$x$ displacement

$\alpha$ tilt angle

$\delta$ minimal length of spring

$\varepsilon$ restitution coefficient

$\varphi_{\mathrm{s}}$ solid fraction of the liquid-solid interface

$\gamma$ surface/interface tension

$\Delta W$ work of adhesion

$\theta$ apparent contact angle

$\theta_{0}$ intrinsic contact angle

$\rho$ density of liquid

$\sigma$ line tension

$\omega$ natural frequency of mass-spring system

$\xi$ surface roughness ratio 


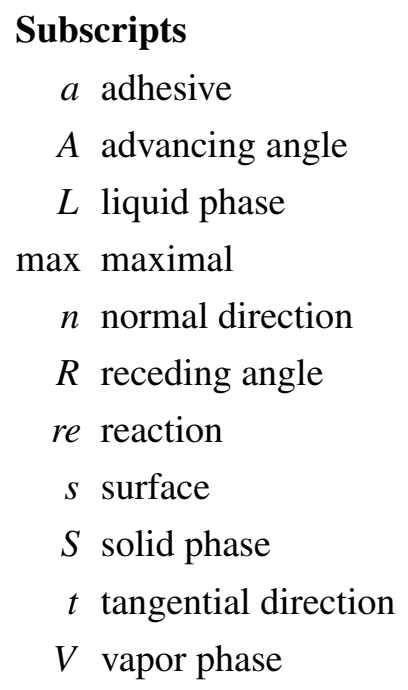

\section{List of Abbreviations}

CA contact angle

CAH contact angle hysteresis

CFHR critical falling height for rebound

MEMS microelectromechanical systems

NEMS nanoelectromechanical systems

PDMS Poly(dimethylsiloxane)

SCCM standard cubic centimeter per minute

$\mathrm{ZnO}$ zinc oxide

\section{Introduction}

A superhydrophobic surface could be utilized to fabricate capillary devices in microelectromechanical systems (MEMS) especially for microfluidic applications $[1,2]$. In such cases, quick transportation of tiny amounts of liquid becomes possible without any leak. In the current decade, the superhydrophobicity and corresponding features have attracted the attention of researchers all over the world [3-7]. Many efforts have focused on the fabrication of superhydrophobic surfaces, and many studies reveal that the static contact angle (CA) and contact angles hysteresis $(\mathrm{CAH})$ are both essential properties for a superhydrophobic surface. Study of impact behavior between a droplet and a surface has drawn much attention. It is found that during the impact process, if the droplet velocity is too small or the CA is not large enough, the droplet would get stuck to the substrate. If the droplet velocity is too large, the droplet would endure extreme deformation during the impact, and finally break into smaller droplets. In a certain velocity range, a more detailed 
analysis of the impact has been carried out by many researchers [7-11]. We have studied the head-on collision of a liquid droplet with another droplet of the same fluid resting on a solid substrate experimentally, and four different responses were observed in the experiments, the contact time was estimated for the complete rebound case by using the Hertz contact model [7]. CA and CAH are both important properties that affect the dynamic response of the droplet. Large CA or limited contact area reduces the adhesion or friction between liquid droplets and solid surfaces, while $\mathrm{CAH}$ occurs due to the wide range of 'metastable' states which can be observed as the liquid meniscus scans the surface of the solid at the solid/liquid/vapor interface $[12,13]$. In this second part, we introduced the origin and influence of $\mathrm{CAH}$ and adhesion number $\left(N_{\mathrm{a}}\right)$, which were essential for the content of this paper.

The exact reasons for $\mathrm{CAH}$ are not known, but there are at least six known sources of CAH [14]. These can be classified into two categories: thermodynamic and kinetic hysteresis. Surface roughness and surface heterogeneity are two sources of thermodynamic hysteresis, which are also two most common of all sources of hysteresis in the natural world. The second classification, kinetic hysteresis, is distinguished by time or cycle dependent changes in the CA. In this category, there are four sources of kinetic CAH: surface orientation, surface deformation, liquid penetration and surface mobility. Detailed descriptions of sources of CAH are summarized in Table 1.

The $\mathrm{CAH}$ is related to the more general phenomenon known as adhesion hysteresis. From the physical viewpoint, the CAH is a measure of energy dissipation during the flow of a droplet [15]. The relation between the adhesion force and the $\mathrm{CAH}$ is:

$$
\frac{F_{\mathrm{a}}}{R}=K \gamma_{\mathrm{LV}}\left(\cos \theta_{\mathrm{R}}-\cos \theta_{\mathrm{A}}\right),
$$

where $F_{\mathrm{a}}$ is the adhesion force, $R$ is the radius of liquid-solid contact area, $K$ is proportionality constant and can be equal to unity [15], $\theta_{\mathrm{R}}$ and $\theta_{\mathrm{A}}$ are receding and advancing angles, respectively. The adhesion number, $N_{\mathrm{a}}$ as the ratio of the adhesion force to the capillary force is defined as [15]:

$$
N_{\mathrm{a}} \cong \frac{\gamma_{\mathrm{LV}}\left(\cos \theta_{\mathrm{R}}-\cos \theta_{\mathrm{A}}\right)}{\gamma_{\mathrm{LV}}}=\cos \theta_{\mathrm{R}}-\cos \theta_{\mathrm{A}} .
$$

The relation between the adhesion number and the work of adhesion is:

$$
N_{\mathrm{a}}=\cos \theta_{\mathrm{A}}-\cos \theta_{\mathrm{R}}=\frac{\Delta W}{\gamma_{\mathrm{LV}}}=\frac{F_{\mathrm{a}}}{R K \gamma_{\mathrm{LV}}},
$$

where $\Delta W$ is the work of adhesion. Thus, the CAH could be taken as an indication of both adhesion and friction. To fully characterize any surface, therefore, it is important to measure both advancing and receding CAs and report the difference as the $\mathrm{CAH}$.

In recent years, in addition to $\mathrm{CA}$ and $\mathrm{CAH}$, the ability of the surface to bounce off droplets has attracted more and more attention. It has been shown that this ability is associated with the energy barrier between Cassie-Baxter and Wenzel states. 
Table 1.

Detailed description of the sources of contact angle hysteresis [14]

\begin{tabular}{|c|c|c|c|c|}
\hline $\begin{array}{l}\text { Sources } \\
\text { of CAH }\end{array}$ & $\begin{array}{l}\text { General } \\
\text { assumption }\end{array}$ & Specific assumption & Effect on hysteresis & $\begin{array}{l}\text { Time } \\
\text { depen- } \\
\text { dent }\end{array}$ \\
\hline \multirow[t]{2}{*}{$\begin{array}{l}\text { Thermody- } \\
\text { namic } \\
\text { hysteresis }\end{array}$} & Smooth surface & $\begin{array}{l}\text { - Surface is smooth at the } \\
0.1 \text { to } 0.5 \mu \mathrm{m} \text { level }\end{array}$ & $\begin{array}{l}\text { - } \theta_{\mathrm{a}} \text { increases and } \theta_{r} \text { de- } \\
\text { creases with increasing } \\
\text { roughness }\end{array}$ & No \\
\hline & $\begin{array}{l}\text { Homogeneous } \\
\text { surface }\end{array}$ & $\begin{array}{l}\text { - Surface is homogeneous } \\
\text { at the } 0.1 \mu \mathrm{m} \text { level and } \\
\text { above }\end{array}$ & $\begin{array}{l}\text { - } \theta_{\mathrm{a}} \text { dependent on low } \\
\text { energy phase } \\
\text { - } \theta_{\mathrm{r}} \text { dependent on high } \\
\text { energy phase }\end{array}$ & No \\
\hline \multirow[t]{4}{*}{$\begin{array}{l}\text { Kinetic } \\
\text { hysteresis }\end{array}$} & $\begin{array}{l}\text { Nondeformable } \\
\text { surface }\end{array}$ & $\begin{array}{l}\text { - Modulus of elasticity } \\
\text { in surface } \gtrsim 3 \times 10^{4} \mathrm{~Pa}\end{array}$ & - Not known & Yes \\
\hline & $\begin{array}{l}\text { Surface does } \\
\text { not reorient }\end{array}$ & $\begin{array}{l}\text { - Reorientation time at time } \\
\text { of measurement }\end{array}$ & $\begin{array}{l}\text { - Increased tendency to } \\
\text { orient lends to increased } \\
\text { hysteresis }\end{array}$ & Yes \\
\hline & $\begin{array}{l}\text { Immobile } \\
\text { surface }\end{array}$ & $\begin{array}{l}\text { - Configuration entropy } \\
\text { independent of local } \\
\text { environment }\end{array}$ & $\begin{array}{l}\text { - Probably increase in hys- } \\
\text { teresis as surface mobility } \\
\text { increases }\end{array}$ & Yes \\
\hline & $\begin{array}{l}\text { Liquid does } \\
\text { not penetrate } \\
\text { surface }\end{array}$ & $\begin{array}{l}\text { - Liquid molecular } \\
\text { volume }>6-7 \times 10^{-2} \\
\mathrm{~m}^{3} / \mathrm{kg} \mathrm{mol}\end{array}$ & $\begin{array}{l}\text { Increased liquid penetra- } \\
\text { tion lends to increased } \\
\text { hysteresis }\end{array}$ & Yes \\
\hline
\end{tabular}

Cassie and Baxter, on the one hand, and Wenzel, on the other hand, determine the apparent CAs of a textured surface. The Cassie-Baxter equation assumes that air pockets are trapped between the droplet and the substrate and the droplet partially sits on the emerging texture. In this case, the apparent CA is determined as follows [16]:

$$
\cos \theta=\varphi_{\mathrm{s}}\left(\cos \theta_{0}+1\right)-1,
$$

where $\varphi_{\mathrm{s}}$ is the solid fraction of the liquid-solid interface, $\theta$ and $\theta_{0}$ are apparent contact angle and intrinsic angle, respectively.

Wenzel equation assumes that the liquid fills all the pores. Under this assumption, the apparent CA equals [17]:

$$
\cos \theta=\xi \cos \theta_{0},
$$

where $\xi$ is the roughness ratio of the surface. From energy considerations, it has been shown that the Cassie-Baxter state is often metastable. In practice, this means that the droplet will remain in a Cassie-Baxter state only if it is subjected to soft external perturbations. Sometimes, the weight of the droplet itself may be sufficient to provoke the impalement. Due to their huge values of $\mathrm{CAH}$, surfaces with Wenzel 
states are not suitable for applications to low-friction droplet displacement or selfcleaning.

In this second part, we discuss the droplet rebound phenomenon on a superhydrophobic surface at low Weber number (We) as well as influence of wettability parameters $\left(\mathrm{CA}, \mathrm{CAH}\right.$ and $N_{\mathrm{a}}$ ). In particular, the critical falling height for rebound (CFHR) has been studied experimentally in the elastic impact (small impact velocity) situation. The theory for normal impact was studied from the viewpoints of spring model. The relationship between tilt angles and CFHR was determined experimentally, and the mechanism of energy dissipation during the impact process was studied. It was found that the adhesion number $N_{\mathrm{a}}$ is the factor governing the energy dissipated by surface tension.

\section{Experimental}

Water CA characterization of $\mathrm{ZnO}$ samples was carried out using an OCA 20 (Dataphysics, Germany) contact angle analyzer and the measurement precision was $\pm 0.1^{\circ}$. For the precise measurement of falling height, the modification of sample stage was necessary. We used the standard sample stage which could vary the tilt angle and the precise lifting platform designed by ourselves was clamped on the standard sample stage. The measurement precision of the lifting platform is $\pm 5 \mu \mathrm{m}$. The schematic diagram is illustrated in Fig. 1(a). The experiments were carried on ZnO-PDMS surface with a static CA of $155.2^{\circ}$ (Fig. 1(b)).

\section{Results and Discussion}

\subsection{Restitution Coefficient}

The restitution coefficient $\varepsilon\left(\varepsilon=\left|V^{\prime} / V\right|\right.$, where $V$ and $V^{\prime}$ are, respectively, the velocities before and after the impact) can be measured for each bounce as shown in Fig. 2. The radius of the droplet is about $1 \mathrm{~mm}$ in the experiments and the falling height ranges from 0.1 to $3 \mathrm{~mm}$. There is a threshold height for rebound (about $0.11 \mathrm{~mm}$ ) and the restitution coefficient increases with the increase of falling height. Then, $\varepsilon$ reaches a maximum $(\varepsilon=0.825)$ and remains independent of the falling height. At still higher impact velocity, $\varepsilon$ slightly decreases with falling height. For a superhydrophobic surface, a stable restitution coefficient is crucial for self-cleaning. Thus, $\varepsilon \geqslant 0.8$ is suggested to be the third criterion for a superhydrophobic surface from the viewpoint of rebounce of droplets from a superhydrophobic surface. Thus, a superhydrophobic surface should meet three conditions: (1) CA $\geqslant 150^{\circ}$; (2) $\mathrm{CAH} \leqslant 10^{\circ}$; and ( 3 ) the restitution coefficient $\varepsilon \geqslant 0.8$.

When the droplet impacts on the surface, it is compressed and the kinetic energy is transferred to surface energy. Then, the droplet retracts while a part of surface energy is transferred to translational kinetic energy (allowing it to take off). Thus, there is a threshold energy (or falling height) for the droplet to take off. At larger falling heights, the restitution coefficient increases gradually, which means that the 


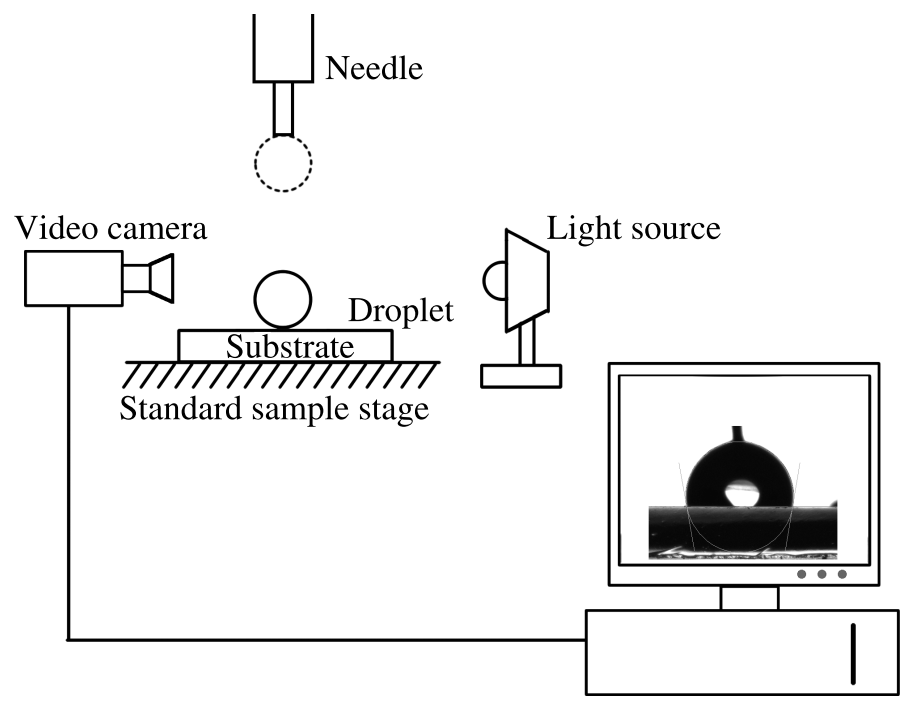

(a)

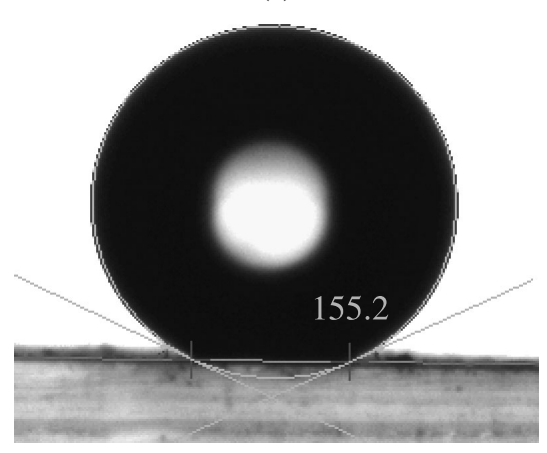

(b)

Figure 1. (a) Schematic diagram of impact of water droplet on ZnO-PDMS surface; (b) static contact angle of the superhydrophobic surface $\left(155.2^{\circ}\right)$.

increment of energy dissipated by viscosity is less than that of translational kinetic energy. Under this condition, a large part of energy could transfer to translational kinetic energy. However, a very large falling height would lead to vigorous liquid motion in the droplet and the energy dissipated by viscosity would increase greatly. So, $\varepsilon$ slightly decreases with falling height at large velocity.

\subsection{Relation between Tilt Angle and Critical Falling Height for Rebound}

Three typical rebound processes are shown in Fig. 3, and the tilt angles are $0^{\circ}, 10^{\circ}$ and $20^{\circ}$, respectively. The pictures show the initial state, maximal compressed state, and taking-off state with three tilt angles. Comparing the last picture for each tilt angle, we can see that the droplet has rolled a little at large tilt angle. The larger the tilt angle is, farther the droplet would move before detachment. The relation between tilt angle and CFHR was studied experimentally. CFHR is defined as the 


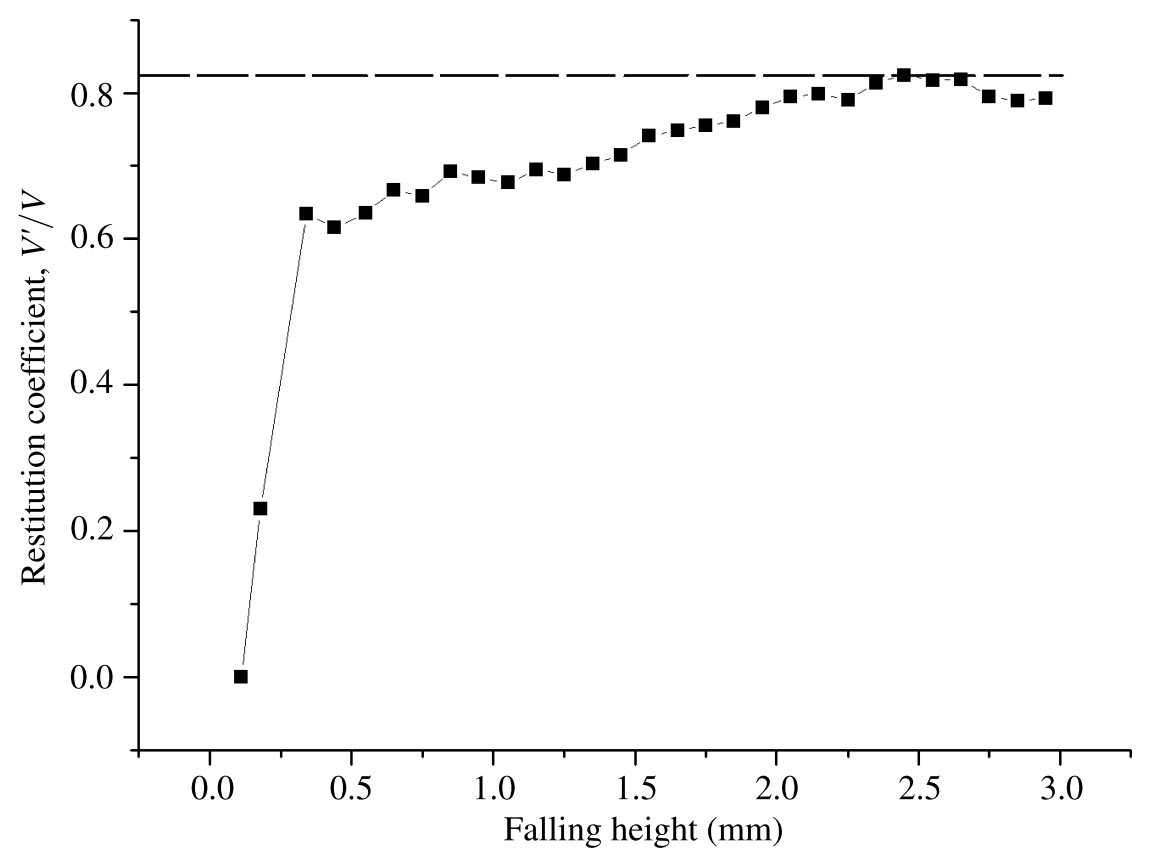

Figure 2. Relationship between falling height and restitution coefficient $\left(V^{\prime} / V\right)$.

falling height at which a full rebound just occurs after impact. If the falling height is smaller than CFHR, the droplet would not detach from the substrate surface. The relation of CFHR with the tilt angle ranging from $0^{\circ}$ to $30^{\circ}$ is shown in Fig. 4. The experiments show that CFHR increases with the increase of tilt angle. This phenomenon could be attributed to the tendency to roll when the tilt angle is large. The critical height ranged from $2.30-2.55 \mathrm{~mm}$ (the distance between droplet and solid surface), which means the impact of rolling was not significant.

Through dimensional analysis, we can obtain the following two dimensionless numbers:

(1) $W e_{\mathrm{n}}$ : representing the ratio of the normal collision energy to the surface energy of the droplet before impact

$$
W e_{\mathrm{n}}=\frac{\rho V_{\mathrm{n}}^{2} r}{\gamma}=\frac{2 g h \rho r \cos ^{2} \alpha}{\gamma}
$$

(2) $W e_{\mathrm{t}}$ : representing the ratio of the tangential collision energy to the surface energy of the droplet before impact

$$
W e_{\mathrm{t}}=\frac{\rho V_{\mathrm{t}}^{2} r}{\gamma}=\frac{2 g h \rho r \sin ^{2} \alpha}{\gamma},
$$

where $W e_{\mathrm{n}}$ and $W e_{\mathrm{t}}$ are normal and tangential Weber numbers, respectively. Subscripts $n$ and $t$ denote the normal and tangential directions of substrate surface. $V_{\mathrm{n}}$ and $V_{\mathrm{t}}$ are normal and tangential velocities of droplet, respectively. $r$ denotes the 


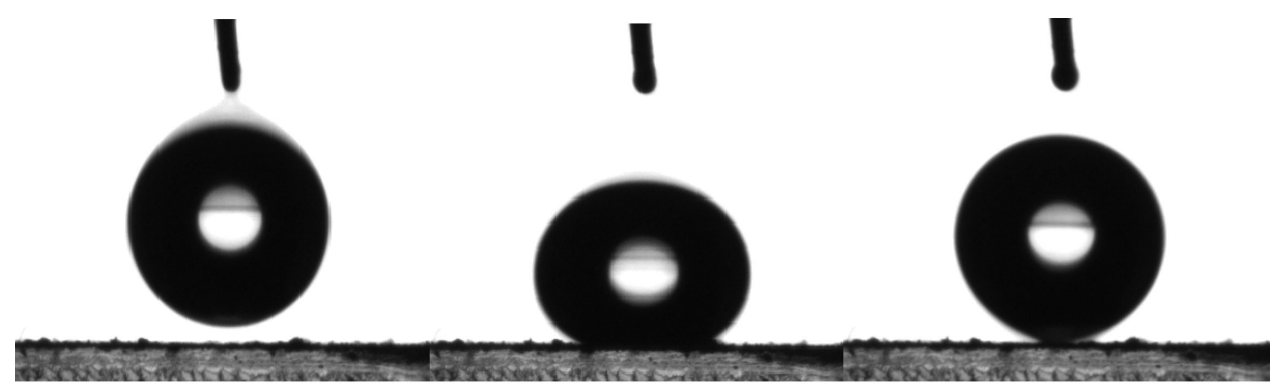

(a)

(b)

(c)

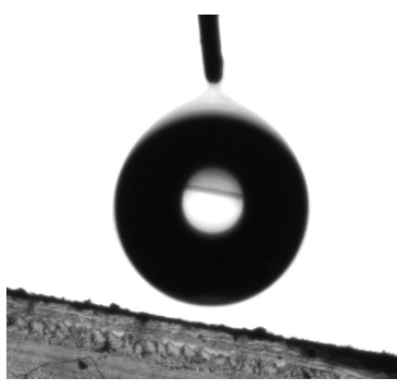

(d)

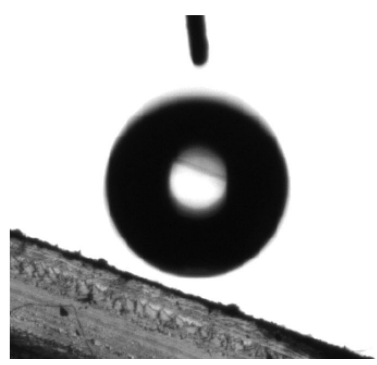

(g)

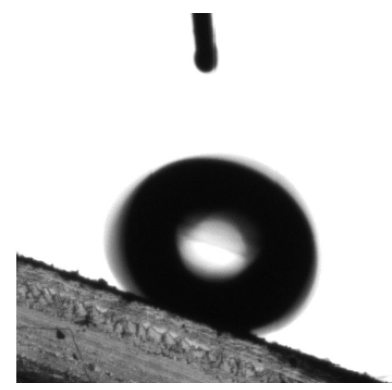

(h)
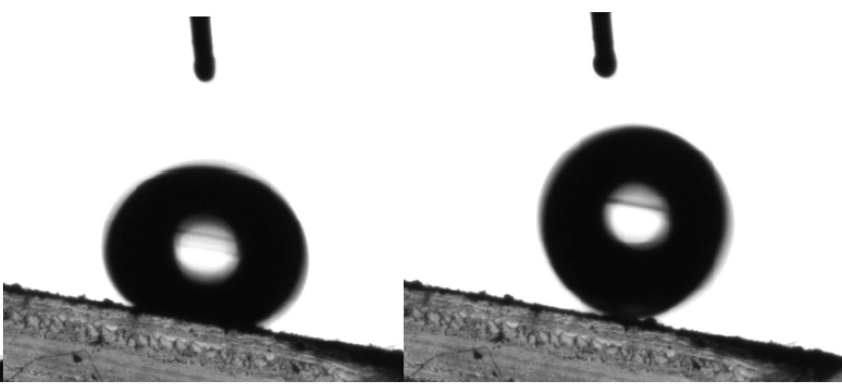

(e)

(f)

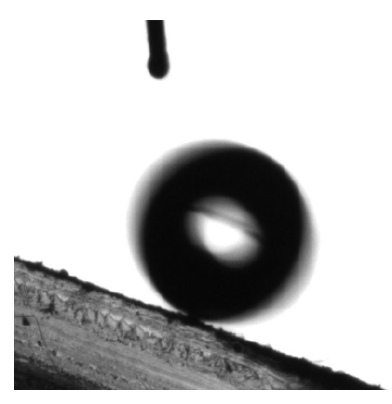

(i)

Figure 3. Rebound processes with different tilt angles: (a-c) tilt angle is $0^{\circ}$; (d-f) tilt angle is $10^{\circ}$; (g-i) tilt angle is $20^{\circ}$.

radius of droplet, and $\gamma$ is the surface tension. It is obvious that a large falling height leads to large $W e_{\mathrm{n}}$ and $W e_{\mathrm{t}}$, and a large tilt angle leads to small $W e_{\mathrm{n}}$ and large $W e_{\mathrm{t}}$ at constant falling height.

The experimental data and standard line which is drawn assuming that the $W e_{\mathrm{n}}$ is the only factor governing the rebound behavior through the following relation are shown in Fig. 4. The equation for the standard line is:

$$
H=\frac{2 \rho g H_{0} d}{\gamma \cos \alpha} .
$$

The origin of the standard line $(0,2.38)$ means that the droplet falling from $2.38 \mathrm{~mm}$ just could detach from surface. It is known that $W e_{\mathrm{n}}$ is an important factor 


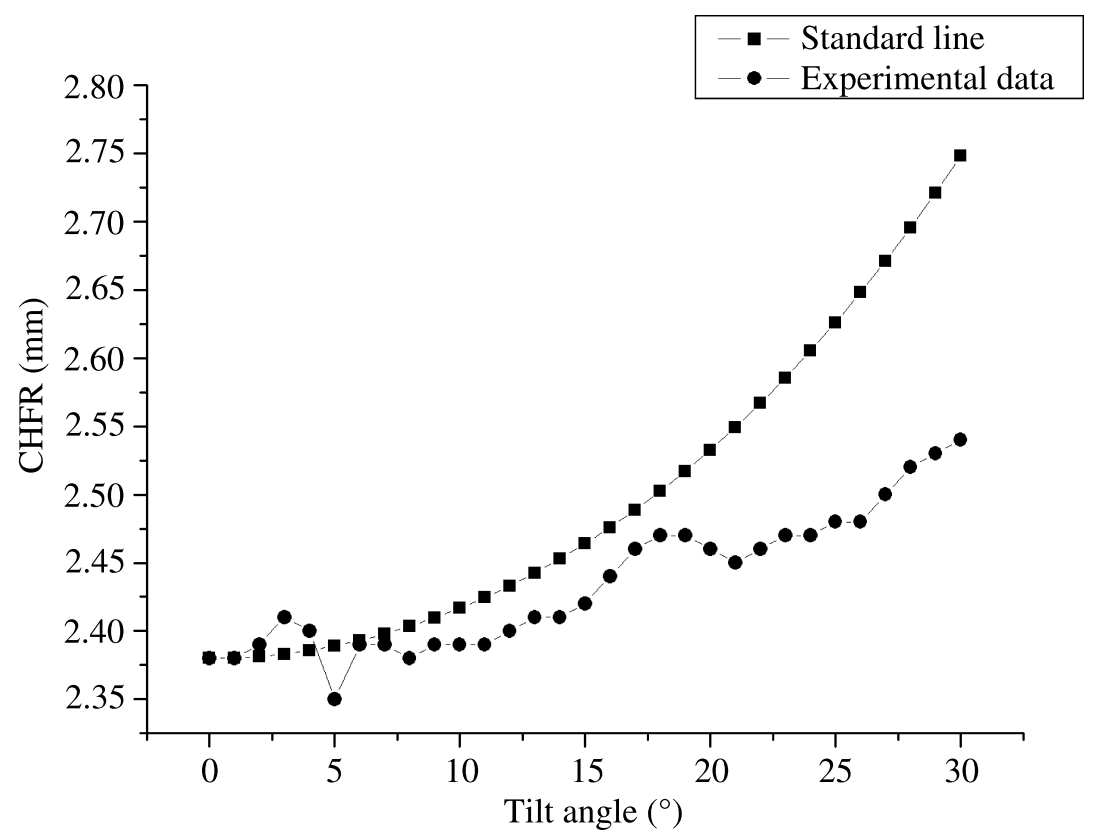

Figure 4. Relationship between CFHR and tilt angle: standard line was drawn according to the formula $H=\frac{2 \rho g H_{0} d}{\gamma \cos \alpha}$.

to the impact behavior. However, the larger the tilt angle is, the larger the discrepancy between standard line and the experiment data will be. These results showed that the influence of $W e_{t}$ on impact process movement cannot be neglected, and rebound was more liable to occur with larger $W e_{\mathrm{t}}$.

\subsection{Theoretical Analysis for Normal Impact}

The droplet rebound phenomenon has been studied by many researchers. Here we propose to model a bouncing droplet as a mass-spring system $[4,11]$. The model is a spring with initial length $l_{0}$ and stiffness $k$ and two masses $m / 2$ attached at each end (Fig. 5). The rebound can be divided into two processes: first, the droplet spreads owing to its kinetic energy, so that it (partially) stores it as surface energy (as a compressed spring does as elastic energy); then, the droplet transfers its surface energy to translational kinetic energy and oscillatory kinetic energy.

Newton's equation of motion can be written for the two masses, whose positions are denoted $x_{1}$ (at the top of the droplet) and $x_{2}$ (at the bottom):

$$
\left\{\begin{array}{l}
\frac{1}{2} m \frac{\mathrm{d}^{2} x_{1}}{\mathrm{~d} t^{2}}=-\frac{1}{2} m g-k\left(x_{1}-x_{2}-l_{0}\right) \\
\frac{1}{2} m \frac{\mathrm{d}^{2} x_{2}}{\mathrm{~d} t^{2}}=-\frac{1}{2} m g-k\left(x_{2}-x_{1}+l_{0}\right)+F_{\mathrm{re}},
\end{array}\right.
$$

where $F_{\text {re }}$ is reaction force of the substrate. 


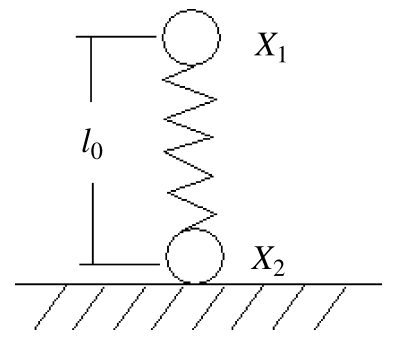

Figure 5. Mass-spring model of droplet: $x_{1}$ and $x_{2}$ denote the displacement of each end.

Initially, and as long as the force acting on mass 2 is negative, $x_{2}=0$, we obtain

$$
x_{1}(t)=l_{0}-\frac{m g}{2 k}+\left(\delta-l_{0}+\frac{m g}{2 k}\right) \cos \omega t,
$$

where $\omega=\sqrt{2 k / m}$ is the natural frequency of the mass-spring system. The bottom mass 2 leaves the substrate if the force which acts on it is positive, i.e.,

$$
k\left(x_{1}-l_{0}\right)-\frac{m g}{2}-F_{\mathrm{a}}>0,
$$

where $F_{\mathrm{a}}$ denotes the adhesion force which is generated from surface tension. $f$ varies during the rebound process, so it is not easy to obtain an exact expression, but the energy needed to overcome the work of adhesion $[18,19]$ is:

$$
W=A \Delta \gamma=A\left(\gamma_{\mathrm{LV}}+\gamma_{\mathrm{SV}}-\gamma_{\mathrm{LS}}\right) \text {, }
$$

where the letters $\mathrm{S}, \mathrm{L}, \mathrm{V}$ denote the solid, liquid and vapor (the surface tension between phases I and $\mathrm{J}$ was denoted by $\gamma_{\mathrm{IJ}}$ ).

For $W e \gg 1, \delta \ll l_{0}$, denoting $x(t)$ as the position of the centre of mass of the spring, the velocity of rebound is:

$$
V^{\prime}=\sqrt{\frac{1}{4} \omega^{2} l_{0}^{2}\left(1-\frac{g}{\omega^{2} l_{0}}\right)\left(1+\frac{3 g}{\omega^{2} l_{0}}\right)-\frac{2 A \Delta \gamma}{m}} .
$$

When $m g \ll k l_{0}$ :

$$
V^{\prime}=\sqrt{\frac{1}{4} \omega^{2} l_{0}^{2}-\frac{2 A \Delta \gamma}{m}} .
$$

For the surface with CA near $180^{\circ}$, we obtain:

$$
V^{\prime}=\frac{1}{2} \omega l_{0} .
$$

Assuming the droplet falls from a height $H$, and the droplet would compress to a point when the diameter of contact area $d_{\max } \sim d_{0} W e^{1 / 4}[9]$

$$
\begin{gathered}
W e=\frac{\rho V^{2} d_{0}}{\gamma_{\mathrm{LV}}}=\frac{2 \rho g H d_{0}}{\gamma_{\mathrm{LV}}}, \\
V^{\prime}=\sqrt{\frac{k}{2 m} l_{0}^{2}\left(1-\frac{m g}{2 k l_{0}}\right)\left(1+\frac{3 m g}{2 k l_{0}}\right)-\frac{\pi \Delta \gamma d_{0}^{2}}{2 m} W e^{1 / 2}},
\end{gathered}
$$


taking $k$ as $\gamma_{\mathrm{LV}}$, and the $l_{0}$ is diameter of droplet, we can determine the velocity of rebound. For the limitations of $\delta \ll l_{0}$ and $m g \ll k l_{0}$, the expression above is valid only for $W e \gg 1$.

\subsection{Energy Dissipation and Rebound Mechanism}

The impact process can be divided into two stages: first, the droplet spreads owing to its kinetic energy, so that it partially stores it as surface energy; then, the droplet transforms its surface energy into translational kinetic energy (allowing it to take off) and oscillatory kinetic energy. Except for the air resistance, the energy of droplet could be dissipated in several ways after the first impact, including translational kinetic energy, oscillating kinetic energy, and energy dissipated by viscosity, energy dissipated by surface tension and inner friction of substrate (dissipated during collision).

When the droplet spreads and retracts on the surface, part of the energy is dissipated by surface tension. First, we describe a simple way to estimate the energy dissipated by surface tension. During the first impact process, the droplet spreads on the superhydrophobic surface. The dynamic CA is considered to be the advancing angle in this process. Thus, the resultant force in horizontal direction does positive work in this process. For the same reason, while the droplet undergoes the retracting process, the dynamic CA is the so-called receding angle. The resultant tension also does positive work. These two processes could consume some part of total energy.

For a static droplet on a solid surface, Young's equation gives:

$$
\gamma_{\mathrm{SV}}=\gamma_{\mathrm{SL}}+\gamma_{\mathrm{LV}} \cos \theta
$$

For the first compressive process, the resultant force per unit length in horizontal direction is:

$$
\sum \gamma_{1}=\gamma_{\mathrm{SV}}-\gamma_{\mathrm{LV}} \cos \theta_{\mathrm{a}}-\gamma_{\mathrm{SL}}=\gamma_{\mathrm{LV}}\left(\cos \theta-\cos \theta_{\mathrm{A}}\right)
$$

For the same reason, the resultant force per unit length in horizontal direction during the retracting process is:

$$
\sum \gamma_{2}=\gamma_{\mathrm{SL}}-\gamma_{\mathrm{SV}}+\gamma_{\mathrm{LV}} \cos \theta_{\mathrm{a}}=\gamma_{\mathrm{LV}}\left(\cos \theta_{\mathrm{R}}-\cos \theta\right)
$$

$\pi>\theta_{\mathrm{A}}>\theta>\theta_{\mathrm{R}}>\pi / 2$, thus, $\sum \gamma_{1}, \gamma_{2}>0$.

During the first process, the droplet spreads owing to its kinetic energy, so that it partially stores it as surface energy. When $W e>1$, the diameter of contact area could be estimated as: $d_{\max } \sim d_{0} W e^{1 / 4}$ [9]. Thus, the contact area is

$$
A=\frac{1}{4} \pi d_{\max }^{2},
$$

where $d_{\max }$ denotes the diameter of the maximal contact area, $d_{0}$ denotes the original diameter of droplet, and We is the Weber number.

The energy dissipated by surface tension is

$$
W=2 A \gamma_{\mathrm{LV}}\left(\cos \theta_{\mathrm{R}}-\cos \theta_{\mathrm{A}}\right) .
$$


The adhesion number, $N_{\mathrm{a}}$, which is an indication of the extent of adhesion with respect to capillary force, is defined as [15]:

$$
N_{\mathrm{a}}=\cos \theta_{\mathrm{R}}-\cos \theta_{\mathrm{A}}=\frac{\Delta W}{\gamma_{\mathrm{LV}}} .
$$

Thus, the energy needed to overcome surface tension can be written as

$$
W=2 A \gamma_{\mathrm{LV}}\left(\cos \theta_{\mathrm{R}}-\cos \theta_{\mathrm{A}}\right)=2 A \Delta W=2 A \gamma_{\mathrm{LV}} N_{\mathrm{a}}
$$

and

$$
\begin{aligned}
N_{\mathrm{a}} & =\cos \theta_{\mathrm{R}}-\cos \theta_{\mathrm{A}} \\
& =-2 \sin \left(\frac{\theta_{\mathrm{R}}+\theta_{\mathrm{A}}}{2}\right) \sin \left(\frac{\theta_{\mathrm{R}}-\theta_{\mathrm{A}}}{2}\right) \approx 2 \sin (\mathrm{CA}) \sin \left(\frac{\mathrm{CAH}}{2}\right) .
\end{aligned}
$$

Generally speaking, a superhydrophobic surface should satisfy two conditions: $\mathrm{CA}>150^{\circ}$ and $\mathrm{CAH}<10^{\circ}$ [20]. Large CA leads to low adhesion and small CAH means low friction. Thus, both large CA and small $\mathrm{CAH}$ are favorable for the rebound of droplet. During the impact process, the energy is dissipated through certain ways. And it has been shown that even in the ideal case of a negligible $\mathrm{CAH}$, the restitution coefficient is found to be significantly lower than unity. One important point is that the droplet oscillates after the lift-off, which means that the surface energy is only partly transferred to the kinetic energy which makes the droplet rebound. Another point is that the energy is largely dissipated by viscosity of the liquid during the impact process.

The total energy could be considered as the gravitational potential energy of the droplet, and translational energy could be calculated from the restitution coefficient. Furthermore, the maximal contact area increases with increasing We (or falling height), and the relationship is shown in Fig. 6. It is found that the relationship could be considered to be linear when $W e$ ranges from 0 to $0.735\left(A_{\max } \approx 2.6 W e+0.77\right)$. Thus, under the assumption of ignoring air resistance, we could calculate the total energy, translational energy, energy dissipated by surface tension, and the sum of oscillating kinetic energy and energy dissipated by water viscosity after the first impact. The relation between total energy, oscillatory kinetic energy and the dissipated energy (collision, viscosity and surface tension) after the first impact are shown in Fig. 7. It shows that the energy is largely dissipated by viscosity, collision and oscillatory kinetic energy, while the influence of substrate surface is not significant. First, the maximal contact area increases with the increase of We as shown in Fig. 6, thus, the energy dissipated by surface tension also increases with increasing We. Second, the impact velocity is directly associated with We, the higher the velocity is, the more disordered the flow field in the droplet will be. Besides, higher velocity (higher $R e$ ) leads to stronger collision, which will consume more energy. For the same reason, the oscillatory kinetic energy also increases with increasing impact velocity. So, corresponding line in Fig. 7 increases with the increase of impact velocity. 


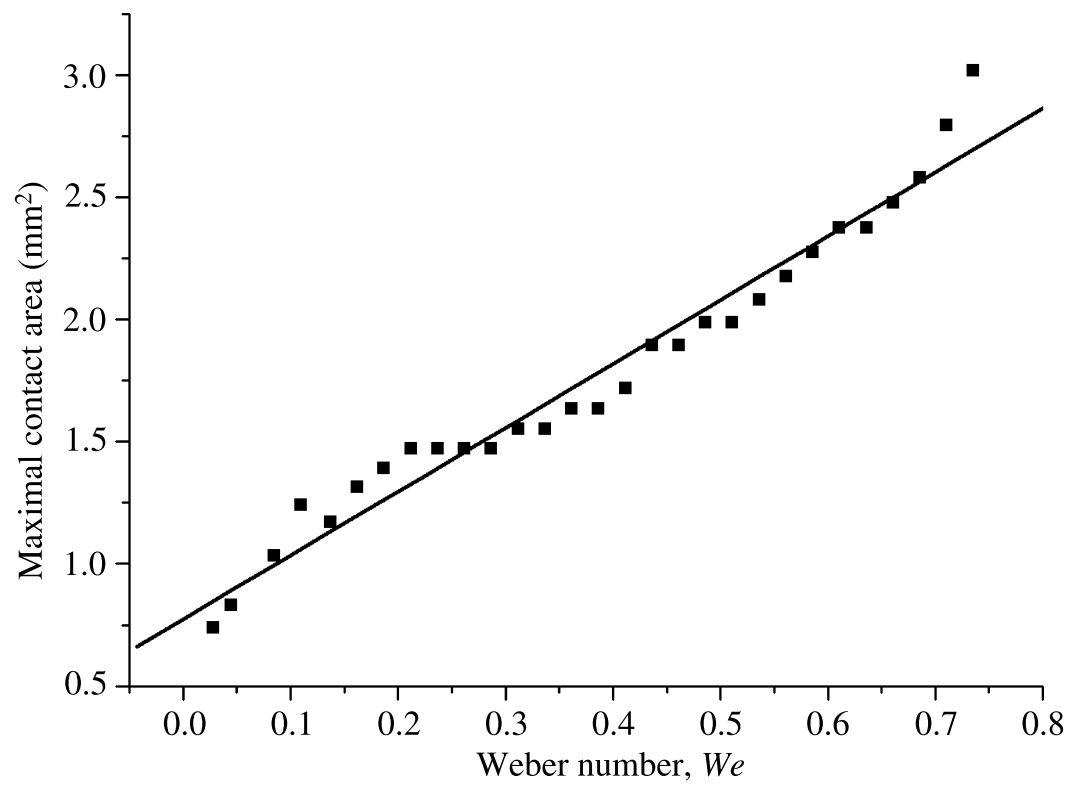

Figure 6. Relationship between We and maximal contact area.

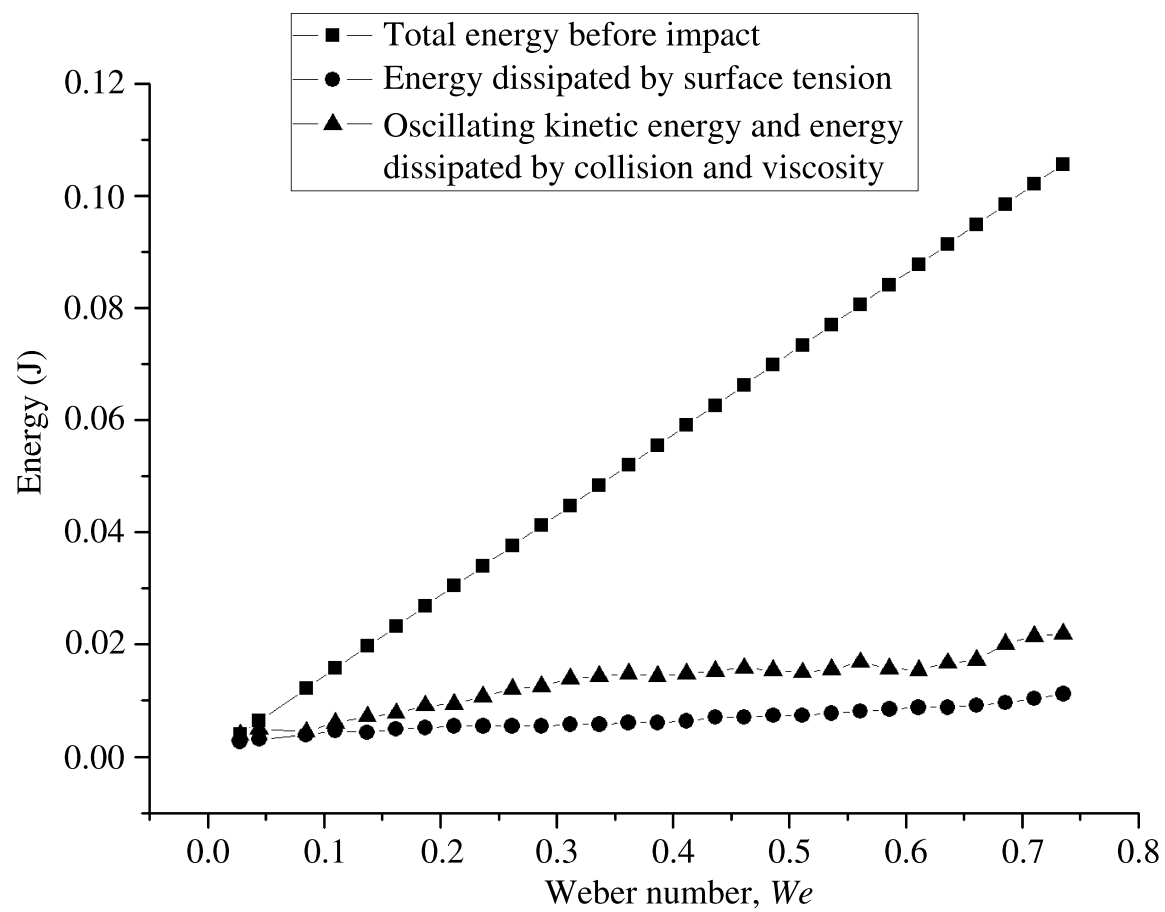

Figure 7. Comparison of total energy and several ways of energy dissipation after the first impact with different $W e$. 
As mentioned in the Section 3.2, We is the governing parameter for the rebound of droplet. However, high We could result in rupture of the droplet, while the energy is not sufficient for the droplet to take off with very low We $(<0.028$ in our experiments). Furthermore, high We could disrupt the thermodynamic balance of surfaces, which means turning wetting state from Cassie to Wenzel. The relatively high adhesion of surface with Wenzel state is not favorable for the rebound of droplet. Viscosity of liquid is another important factor. In order to understand the influence of viscosity, two more dimensionless numbers, capillary number $(\mathrm{Ca})$

$$
C a=\frac{\mu_{\mathrm{L}} V}{\gamma_{\mathrm{LV}}}
$$

and Reynolds number $(R e)$

$$
R e=\frac{\rho V r}{\mu_{\mathrm{L}}},
$$

should be introduced. Capillary number and Reynolds number characterize the ratio of the viscous to capillary force and the ratio of inertial to viscous force, respectively. High viscosity (high $\mathrm{Ca}$ and $\mathrm{Re}$ ) would result in dissipation and eventual wetting of the surface.

\section{Conclusions}

The impact behavior of a water droplet on a superhydrophobic ZnO-PDMS surface has been studied both experimentally and theoretically. The restitution coefficient was found to be independent of falling height in a certain range. Thus, we suggest $\varepsilon \geqslant 0.8$ to be the third criterion for a superhydrophobic surface. Oblique impact experiments were carried with the tilt angle ranging from $0^{\circ}$ to $30^{\circ}$. The CFHR increases with the increase of tilt angle, which reveals that the $W e_{\mathrm{n}}$ is the key factor governing the rebound behavior. However, the influence of $W e_{\mathrm{t}}$ on impact behavior cannot be neglected, and a larger $W e_{\mathrm{t}}$ makes rebound much easier. The energy dissipated due to surface properties was small compared to the energy dissipated by viscosity and collision. Besides, $N_{\mathrm{a}}$ is the parameter governing the energy dissipation due to surface tension, which means that only large CA or small CAH is not sufficient for a droplet to bounce off.

\section{Acknowledgements}

This work was jointly supported by the National High-Tech R\&D Program of China (863 Program, Grant No. 2007AA021803), National Basic Research Program of China (973 Program, Grant No. 2007CB310500), and National Natural Science Foundation of China (NSFC, Grant Nos 10772180 and 10721202).

\section{References}

1. L. Zhai, F. C. Cebeci, R. E. Cohen and M. F. Rubner, Nano Lett. 4, 1349 (2004).

2. C. Journet, S. Moulinet, C. Ybert, S. T. Purcell and L. Bocquet, Europhys. Lett. 71, 104 (2005). 
3. A. Carré and K. L. Mittal (Eds), Superhydrophobic Surfaces, VSP/Brill, Leiden (2009).

4. K. Okumura, F. Chevy, D. Richard, D. Quere and C. Clanet, Europhys. Lett. 62, 237 (2003).

5. S. L. Ren, S. R. Yang and Y. P. Zhao, Langmuir 20, 3601 (2004).

6. R. Kannan and D. Sivakumar, Colloids Surfaces A 317, 694 (2008).

7. F. C. Wang, J. T. Feng and Y. P. Zhao, J. Colloid Interface Sci. 326, 196 (2008).

8. J. T. Feng, F. C. Wang and Y. P. Zhao, Biomicrofluidics 3, 022406 (2009).

9. D. Richard and D. Quere, Europhys. Lett. 50, 769 (2000).

10. C. Clanet, C. Beguin and D. Richars, J. Fluid Mech. 517, 199 (2004).

11. A. L. Biance, F. Chevy, C. Clanet, G. Lagubeau and D. Quere, J. Fluid Mech. 554, 47 (2006).

12. B. B. Wang, J. T. Feng, Y. P. Zhao and T. X. Yu, J. Adhesion Sci. Technol., accepted.

13. A. Lafuma and D. Quere, Nature Mater. 2, 457 (2003).

14. J. D. Andrade, L. M. Smith and D. E. Gregonis, in: Surface and Interfacial Aspects of Biomedical Polymers, J. D. Andrade (Ed.), pp. 249-292. Plenum, New York, NY (1985).

15. D. N. Rao, in: Contact Angle, Wettability and Adhesion, K. L. Mittal (Ed.), vol. 3, pp. 191-210. VSP, Utrecht (2003).

16. A. B. D. Cassie and S. Baxter, Trans. Faraday Soc. 44, 11 (1948).

17. R. N. Wenzel, Ind. Eng. Chem. 28, 988 (1936).

18. S. Wu, Polymer Interface and Adhesion. Marcel Dekker, New York, NY (1982).

19. D. Oner and T. J. McCarthy, Langmuir 16, 7777 (2000).

20. B. Balu, V. Breedveld and D. W. Hess, Langmuir 24, 4785 (2008). 